\title{
Impairment Evaluation of Trigeminal Nerve Injury
}

\author{
Kyung-Gyun Hwang \\ Committee on the Evaluation for Maxillofacial Impairment, \\ The Korean Association of Oral and Maxillofacial Surgeons, Seoul, Korea
}

\section{Introduction}

Paresthesia caused by damage of the inferior alveolar nerve and mental nerve as branches of the trigeminal nerve is a common neurological damage in dentistry related to the extraction of mandibular third molar and implant placement. In America, the occurrence rate of paresthesia in the inferior alveolar nerve related to the extraction of mandibular third molar was $0.4 \sim 8.4 \%$. In 2009, a domestic research reported a rate of $0.14 \sim 0.19 \%$. For the impairment evaluation on the damage of the inferior alveolar nerve, McBride impairment evaluation is generally used. Written by Professor McBride in 1963, this standard was out of print after the 6th edition was published in $1963^{1)}$. Note, however, that medicine has developed drastically after the 1930s when McBride's standard was established, yet the McBride standard does not reflect such changes. The reasons are improvement of modern medicine, impairment evaluation and impairment treatment, development of new treatments, and absence of a revised edition for 50 years. In Korea, McBride's standard has been used frequently to determine the rate of physical impairment. In addition to the McBride impairment evaluation, the standard of the State Tort Liability Act - which is used occasionally to evaluate the rate of impairment caused by local neurological damage - was derived from Japan's compensation and impairment system in past. It is irrational because the influence of occupational type is not reflected at all, the detailed items for evaluating impairment in the maxillofacial area including teeth are insufficient, the rate of impairment is abstract, and the difference in loss rate between rates is too large. In particular, the concept of implant as frequently used in dental clinics nowadays is not reflected; hence the difficulty in regarding as a rational impairment evaluation the standard of "calculating the loss rate of labor capacity after teeth loss as 5\% uniformly" based on the number of dentures, e.g., paresthesia.

Objective and rational impairment evaluation is necessary given the increasing medical disputes. In particular, when evaluating the impairment rate due to damage of the inferior alveolar nerve (branch of trigeminal nerve) as a common reason for medical dispute, it is rational to determine the physical impairment rate in oral and maxillofacial surgery based on the standard of the American 
Medical Association (AMA) - the most scientific and reputable standard - and the standard of the American Association of Oral and Maxillofacial Surgeons (AAOMS). Therefore, the Korean Association of Oral and Maxillofacial Surgeons and committee on the evaluation for maxillofacial impairment reviewed the existing standards of impairment evaluation and standards for physical impairment by the AMA and AAOMS and suggested a more rational, applicable impairment evaluation guideline.

\section{Impairment Evaluation}

Impairment evaluation refers to the evaluation of the level of impairment caused by damage due to disease. In the standard for impairment evaluation by the Korean Academy of Medical Sciences (KAMS), it must be conducted based on scientific basis, objectivity, convenience, rationality, and practicality. Evaluation is done when the symptom is stabilized, with re-evaluation required after 2 years if the symptom is expected to change. Currently, there are many impairment evaluation standards, but the most universal impairment evaluation standards are the McBride impairment evaluation, AMA impairment evaluation, impairment evaluation based on the State Tort Liability Act, KAMS impairment evaluation, and AAOMS guideline.

\section{Impairment Rate and Loss Rate of Labor Capacity}

The physical impairment rate and loss rate of labor capacity are derived from the concepts of World Health Organization (WHO) in 1980. The impairment rate seeks to evaluate the level of physical impairment and functional deterioration, whereas the loss rate of labor capacity involves evaluating economic or social damage. Currently, the State Tort Liability Act, McBride standard, and AMA standard are commonly used for impairment rate evaluation. In 2011, KAMS suggested its own standard by revising the AMA standard for Korean society. The loss rate of labor capacity is determined based on the physical impairment rate and various factors including age, gender, job, and education level.

\section{Damage of the Trigeminal Nerve and Impair- ment Rate}

\section{1) Trigeminal Nerve}

Trigeminal nerve is the 5th cerebral nerve and a mixed nerve consisting of sensory fiber that manages the senses in skin and mucous membrane of the head and motor fiber that manages the masticatory muscle. The main branches of the trigeminal nerve are ophthalmic nerve, maxillary nerve and mandibular nerve. Most of the trigeminal nerve is sensory nerve, whereas the mandibular nerve partly consists of motor fiber.

2) Calculation of Physical Impairment Rate and Loss Rate of Labor Capacity Caused by Damage of the Inferior Alveolar Nerve

(1) 5th AMA Impairment Evaluation Standard $(2000)^{2}$ : Evaluation of the senses is conducted by comparing the sense of pain, heat, and touch or both sides of the face. Paresthesia caused by damage of the unilateral mandibular nerve is calculated by applying half (unilateral) and $1 / 3$ (only for mandibular nerve) of the total impairment rate of the trigeminal nerve. The impairment rate may be $0 \sim 2.3 \%$ for Class I $(0 \sim 14 \%), 2.5 \sim 4 \%$ for Class II (15 24\%), and 4.2 5.8\% for Class III (25 35\%). The impairment rate may be $0 \sim 2.3 \%$ in case of paralysis of the lower lip only, $2.5 \sim 4 \%$ if there is moderate facial pain, and $4.2 \sim 5.8 \%$ if there is severe facial pain.

(2) AAOMS' Guideline ${ }^{3)}$ : An impairment evaluation standard related to the oral and maxillofacial area was suggested in 2008 to evaluate the impairment of facial neuropathic pain combined with 
paralysis related to damage of the inferior alveolar nerve. It does not have standards related to unilateral application, however. Thus, if half of the impairment standard related to the ambilateral trigeminal nerve is applied, the impairment rate may be $1.5 \sim 2.5 \%$ for light and moderate impairments combined with uncontrolled facial neuropathic pain and 3 5\% for severe impairments combined with uncontrolled facial neuropathic pain.

\section{Conclusion}

After reviewing the various impairment evaluation standards applied to paresthesia, anesthesia, and facial neuropathic pain caused by damage of the mandibular nerve, I suggest the following guideline for more objective and fair impairment evaluation:

1) The time of impairment evaluation is at least 2 years after the occurrence of symptom due to damage of the inferior alveolar nerve.

2) Considering the various standards, for the evaluation of the physical impairment rate, the impairment rate should be $0 \sim 5 \%$ for general impairment. The $0 \sim 5 \%$ impairment section should be divided as follows:

\begin{tabular}{lcc}
\hline & $\begin{array}{c}\text { Loss and deterioration of } \\
\text { sense }\end{array}$ & Facial neuralgia \\
\hline Light & $0 \sim 1 \%$ & $1 \sim 2 \%$ \\
Moderate & $1 \sim 2 \%$ & $2 \sim 3 \%$ \\
High & $2 \sim 2.5 \%$ & $3 \sim 5 \%$ \\
\hline
\end{tabular}

3) For a more objective evaluation of the aforesaid standard, there is a need to conduct research on the scientific and objective evaluation of paresthesia and pain of lower lip.

\section{References}

1. McBride ED: Disability evaluation and principles of treatment of compensable injuries. 6th ed. Philadelphia: Lippincott Company; 1963.

2. Cocchiarella L, Andersson GBJ: Guides to the evaluation of permanent impairment. 5th ed. Chicago: American Medical Association; 2001.

3. American Assoicateion of Oral and Maxillo Facial Surgeions. Guidelines to the evaluation of impairment of the oral and maxillofacial region. Rosemont: American Association of Oral and Maxillofacial Surgeons; 2008. 